\title{
A SINGLE-CHIP CMOS-MEMS MICROGRAVITY ACCELEROMETER
}

\author{
Y. Zhang ${ }^{*}$, G. Meng, and N. Yazdi \\ Evigia Systems, Inc., Ann Arbor, Michigan, USA
}

\begin{abstract}
This paper reports a single-chip CMOS-MEMS microgravity accelerometer for precision acceleration measurements at micro-g levels. This sensor employs a novel four-quadrant symmetric device structure with full-bridge capacitive output, and a robust fabrication process to form thick single-crystalline silicon sensors atop of CMOS wafers providing high-sensitivity, low-noise and high-stability. The accelerometer is sealed at wafer-level using silicon-caps to lower drift and shield EMI. It has a sensitivity of $1.8 \mathrm{~V} / \mathrm{g}$ and noise floor of $1.2 \mu \mathrm{g} / \mathrm{rt}-\mathrm{Hz}$.
\end{abstract}

\section{INTRODUCTION}

Capacitive micro-accelerometers [1] have advantages of high sensitivity, good dc response, low noise, low drift, low temperature sensitivity, low-power dissipation, and large readout bandwidth. High-sensitivity in the capacitive accelerometer designs, including both combined surface-bulk micro-machined accelerometers [2] and SOI devices [3], is typically attained by using larger lateral dimensions and thick proof-masses in sensor structures. These devices are hybrid packaged with the interface circuitry resulting in larger parasitic, lower electrical signal-to-noise ratio, and EMI susceptibility through the sensor/circuit interconnects. Surface micro-machined accelerometers [4] have the advantages of monolithic integration with the interface circuit. The proof-mass is however small, resulting in a lower mechanical sensitivity and typically 10 's-100's of micro-g mechanical noise floor.

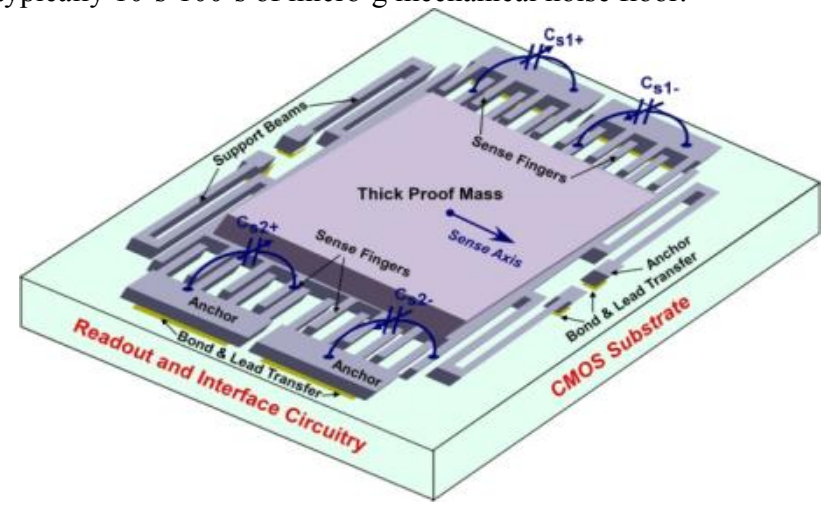

Figure 1: An integrated microgravity accelerometer and low-noise CMOS interface circuitry. The sensor fabrication is completed after integration with the CMOS wafer.

In this paper, we present a novel fully-symmetric highsensitivity silicon accelerometer integrated with the interface CMOS circuitry. Figure 1 shows the overall CMOS-MEMS accelerometer device structure. It is a lateral accelerometer with $50-100 \mu \mathrm{m}$ thick interdigitated fingers and $3-5 \mu \mathrm{m}$ air gap. The sensor achieves high-sensitivity and low-noise via use of thick single-crystalline structure and low-parasitic CMOS integration. Mechanical and temperature stability is improved by using a fullydifferential four-quadrant symmetric sense and readout. The sensor structure is fabricated on a SOI wafer. It uniquely forms a four-quadrant symmetric capacitive full-bridge with isolated sense capacitor leads, which enables the formation of a full-bridge electronic readout without any reference capacitors. The sensing fingers are formed by front-side high aspect ratio etching of the device layer. The handle layer of the SOI wafer is used to define a thick proof mass. The sensor wafer is directly integrated with a CMOS wafer that makes multiple low-parasitic local leads between sensors and circuits possible. This wafer-level integration also simplifies the final sensor package, thus further reduces the manufacturing cost. The sensors and the IC circuitry are hermetically packaged at the wafer level. This level of sensor package protects the sensors from ambient and also serves as EMI shield.

\section{SENSOR DESIGN}

The proper design of MEMS sensors is primarily important in accomplishing good sensor performance. The major design goal is to achieve high sensitivity, low mechanical noise-floor, low crossaxis sensitivity and low temperature sensitivity while minimizing the overall sensor size and maintaining a high-yield fabrication process. This microgravity accelerometer uses capacitive sensing scheme due to its good dc-response, high-sensitivity, low-noise and low-temperature drift. The static open-loop sensitivity of a capacitive accelerometer is:

$$
\frac{\Delta C_{\text {static }}}{a}=N \frac{\varepsilon_{0} A m}{g_{s}^{2} k}=N \frac{\varepsilon_{0} A}{g_{s}^{2} \omega_{r}^{2}}
$$

where $\Delta C_{\text {static }}$ is the sensor capacitance change, $a$ is the input acceleration, $m$ is the proof mass, $k$ is the suspension spring constant, $A$ is the sense area per electrode and $N$ is the number of electrodes, $g_{s}$ is the air gap, $\omega_{r}$ is the mechanical self-resonance frequency, and $\varepsilon_{0}$ is the air permittivity. Mechanical noise is caused by the Brownian motion of the gas molecules surrounding the proof mass and the Brownian motion of the proof mass suspension or anchors. The Total Noise Equivalent Acceleration (TNEA rms- $\left[\mathrm{m} / \mathrm{sec}^{2} / \sqrt{\mathrm{Hz}}\right]$ ) can be calculated by

$$
T N E A=\frac{\sqrt{4 K_{B} T D}}{m}=\sqrt{\frac{4 K_{B} T \omega_{r}}{Q m}}
$$

where $K_{B}$ is the Boltzmann constant and $T$ is the temperature in Kelvin, $D$ is the damping factor, and $Q$ is the quality factor. For the parallel plates that are not at low-pressure ambient and their width and length are significantly larger than the air gap, squeeze film damping is the dominant regime. The squeeze film damping can be calculated based on the geometries and the surrounding gas viscosity $(\mu)$. For instance, the damping factor $D$ for $N$ interdigitated fingers with length of $L$ and thickness of $T$, separated by gap of $g_{s}$ is given by:

$$
D=0.427 N \mu \frac{T^{3} L}{g_{s}^{3}}=0.427 N \mu \frac{A T^{2}}{g_{s}^{3}}
$$

Equations (1)-(3) indicate that the target high-performance of the microgravity accelerometer requires formation of thick large mass, soft suspension, narrow air gap over a large area, and small damping simultaneously.

\section{Sensor Device Structure}

The sensor structure is designed to be fabricated on a SOI wafer and integrated with the CMOS interface circuit at wafer 
level. As shown in Fig. 2, each accelerometer has four sets of interdigitated sensing finger pairs and each finger pair has one moving finger and one fixed finger to form a parallel-plate capacitor. The moving fingers are attached to the proof mass while the fixed fingers are anchored to substrate. The sensing fingers are formed by front-side high aspect ratio etching in the device layer, which will allow formation of $2-5 \mu \mathrm{m}$ air gap with $50-100 \mu \mathrm{m}$ finger thickness. The finger gaps are asymmetrical $\left(\mathrm{g}_{0}>\mathrm{g}_{\mathrm{s}}\right)$ to provide a net capacitance change when the proof mass displaces with respect to the fixed fingers under presence of acceleration. For $\mathrm{g}_{0}>4 \mathrm{~g}_{\mathrm{s}}$ the capacitance sensitivity is reduced only $\sim 6 \%$ compared with the case where the sense element is formed by two parallel plates separated by a gap of $g_{s}$. The thick handle layer of the SOI wafer is used to define a thick proof mass $(200-400 \mu \mathrm{m}$ thick), and the proof mass is supported by four folded and suspended support beams. Because of the usage of the thick handle layer as the proof mass and the design of the folded support beams as the soft suspension, a high sensitivity is obtained while the overall sensor size is much reduced.
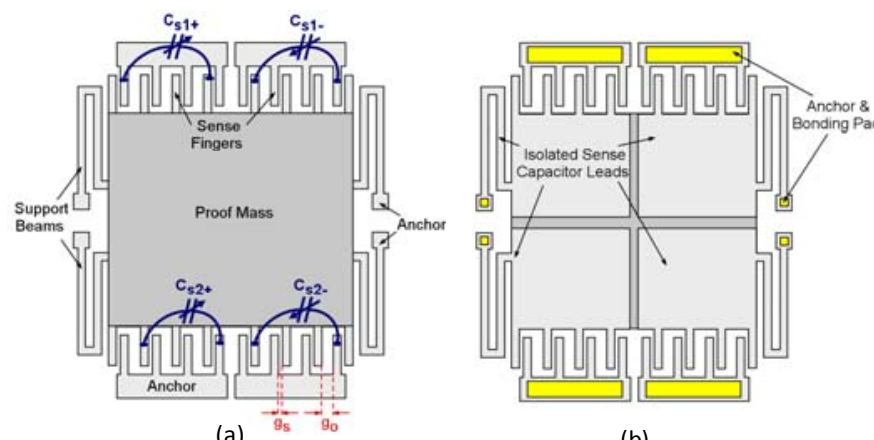

(a)

(b)

Figure 2: A microgravity accelerometer sensing element: (a) a top view and (b) a bottom view.

The high capacitive sensitivity and low mechanical noise are achieved by a symmetric capacitive full-bridge structure to directly interface with a fully-differential readout front-end without any need for reference capacitors. The full-bridge structure improves the overall sensor module sensitivity and lowers drift and temperature sensitivity. In general, this sensor-readout approach is not possible with many commonly used MEMS accelerometer structures in which the proof mass forms a common sensecapacitance node. In this design, however, the device layer and the handle layer are electrically isolated from each other, and its four electrodes of the sense capacitive bridge are isolated at the same step as the sensing finger definition, as shown in Fig. 2(b), such that it makes a capacitive full-bridge possible.

\section{Sensor Geometrical Design and Noise Performance}

The overall sensor design is driven by minimizing the combined electrical and mechanical noise in the smallest die size. Figure 3 illustrates a simulated noise performance with respect to the sensing air gap in the designed accelerometer with two different proof mass thicknesses of $250 \mu \mathrm{m}$ and $350 \mu \mathrm{m}$. The length, thickness, and separation of the interdigitated fingers are $180 \mu \mathrm{m}, 80 \mu \mathrm{m}$, and $7 \mu \mathrm{m}$, respectively, in the simulation. Based on the simulation results, an air gap of $4 \mu \mathrm{m}$ is selected, which results in a lower noise-floor of $0.32 \mu \mathrm{g}-\mathrm{rms} / \sqrt{\mathrm{Hz}}$ with $350 \mu \mathrm{m}$ proof mass, and $0.45 \mu \mathrm{g}-\mathrm{rms} / \sqrt{\mathrm{Hz}}$ with $250 \mu \mathrm{m}$ proof mass at 1 atmosphere ambient pressure.

Table 1 presents the summary of the sensor design parameters. The cross axis sensitivity was analyzed by means of

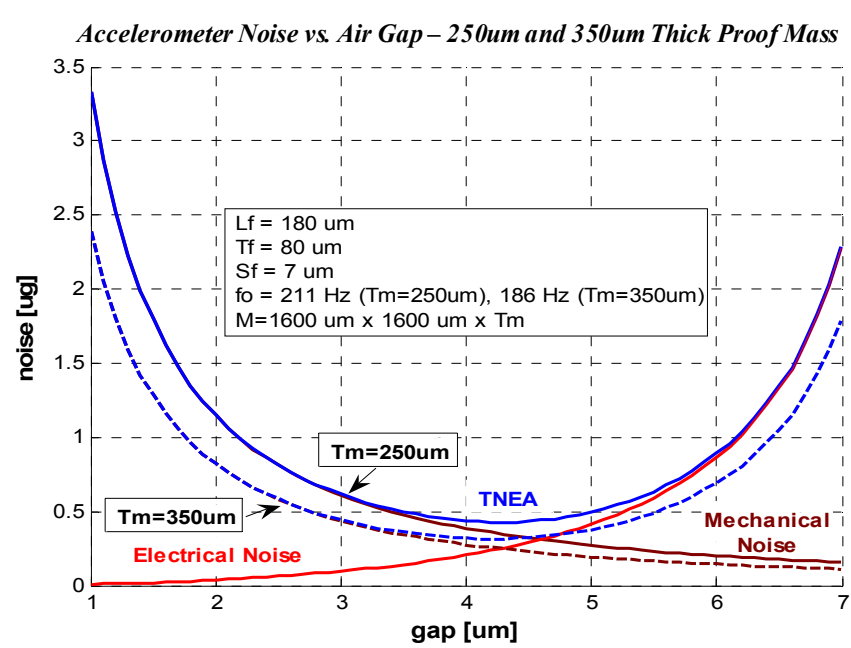

Figure 3: The rms-noise of the designed accelerometer with a lateral dimension of $1600 \mu \mathrm{m} \times 1600 \mu \mathrm{m}$ and thickness of $250 \mu \mathrm{m}$ and $350 \mu \mathrm{m}$ of proof mass. The air gap of $4 \mu \mathrm{m}$ is selected.

FEM simulations and comparisons of sensor structure frequency modes. The $\mathrm{z}$-axis mode is the $2^{\text {nd }}$ mode and mostly impacting the cross-axis sensitivity. The cross-axis sensitivity is minimized by the proper geometrical design of the support beams, by reducing the distance of proof mass gravity center to the support beam plane, and by optimizing the ratio of the proof mass thickness to the beam height. In addition, the four-quadrant symmetrical device structure helps with reducing the cross axis sensitivity. The sensor $\mathrm{z}$-axis cross axis sensitivity is less than $0.3 \%$, and the $y$-axis cross axis is about an order of magnitude smaller.

Table 1: Summary of the accelerometer design parameters.

\begin{tabular}{|l|l|}
\hline Design Parameter & Value \\
\hline Sense fingers & \\
Sense gap & $4 \mu \mathrm{m}$ \\
Length & $180 \mu \mathrm{m}$ \\
Thickness & $80 \mu \mathrm{m}$ \\
Width & $5 \mu \mathrm{m}$ \\
Separation gap & $8 \mu \mathrm{m}$ \\
Number & $4 \times 36$ \\
\hline Proof mass & $1600 \mu \mathrm{m} \times 1600 \mu \mathrm{m} \times 350 \mu \mathrm{m}$ \\
\hline Resonance frequency & $186 \mathrm{~Hz}$ \\
\hline Cross-axis sensitivity & better than $0.3 \%$ \\
\hline Rest capacitance & $4 \times 1.2 \mathrm{pF}$ \\
\hline Capacitive sensitivity & $4 \times 1.7 \mathrm{pF} / \mathrm{g}$ \\
\hline Simulated TNEA & $<0.4 \mu \mathrm{g}-\mathrm{rms} / \mathrm{rt}-\mathrm{Hz}$ \\
\hline
\end{tabular}

\section{Sensor Stability}

The mechanical and temperature stability is obtained by constructing sensor structures with all single-crystal silicon which has excellent stable mechanical characteristics resulting in higher sensor mechanical stability and minimum temperature related stress variations. The bonding pads at the anchors are formed by metal bond. However, these metal pads will not impact the device performance adversely because they are connected to the substrate rigidly. Furthermore the symmetrical full-bridge structure also helps to lower any potential residual effects and to cancel temperature effect as well. In addition, the high stability over wide temperature range will be achieved by employing folded-beam suspensions with which any residual thermo-mechanical stresses induced by the CMOS dielectric and metal layers can be absorbed. 


\section{SENSOR FABRICATION AND INTEGRATION}

The sensor fabrication process is fully compatible with postCMOS processing, and enables formation of the designed sensor structure and its CMOS integration. All fabrication steps are processed in batch and performed at wafer-level. The combination of the steps is a high-yield process, which can be performed in a standard MEMS fabrication facility. All these features provide a clear competitive advantage for accelerometer manufacturing. The fabrication, integration and packaging consist of four parts as shown in Fig. 4.
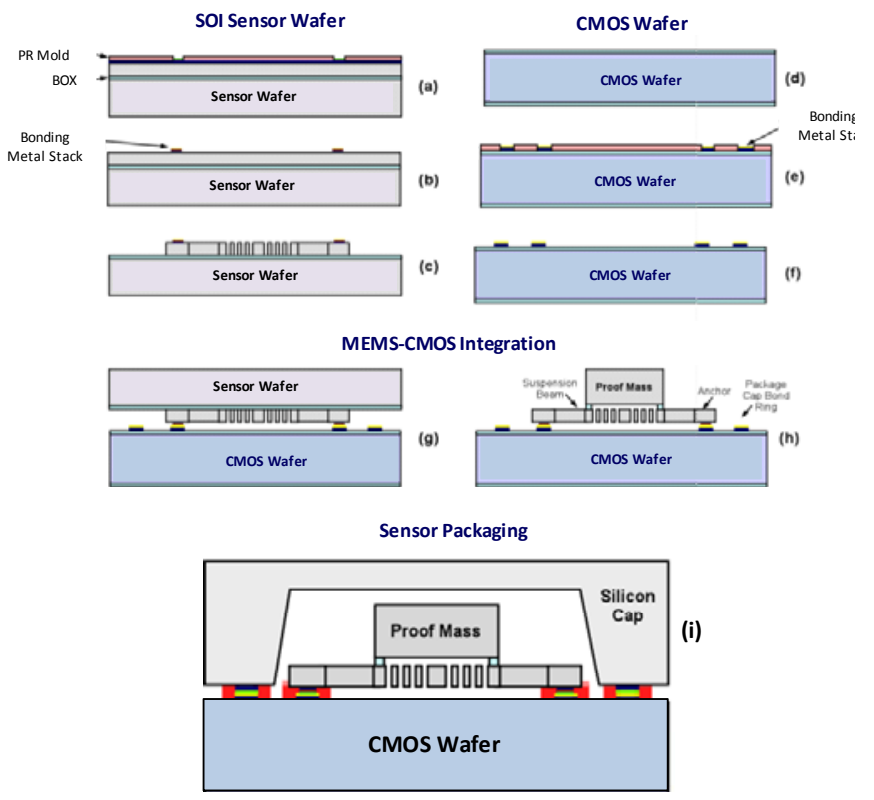

Figure 4: CMOS-MEMS microgravity accelerometer fabrication, integration and packaging process flow.

The first part includes two masking steps performed on a SOI wafer with $50-100 \mu \mathrm{m}$ device layer thickness and $200-400 \mu \mathrm{m}$ thick handle layer. The process starts with electroplating the bonding metal stack on the device layer using a photo resist mold (Fig. 4(a)). The plating seed layer and photo resist mold are removed after electroplating (Fig. 4(b)). It is followed by patterning and DRIE etching on device layer to define sensing fingers, anchors, and suspension support beams. The etch mask is removed to complete the first part of the process (Fig. 4(c)).

The second part of the process has one masking steps and is performed on the CMOS wafers (Fig. 4(d)) containing the interface circuitry. $\mathrm{Cr} / \mathrm{Au}$ metal layers are deposited and patterned for bonding pads (Fig. 4(e)). These pads are in contact the CMOS pads and transfer the leads from the CMOS circuits to the sensors. Metal bonding ring for sensor cap package can also be defined at this step.

The third part of the process includes a wafer bonding and a single masking step for proof mass etching after wafer bonding. The fabricated sensor wafer is flipped, aligned to the bonding pads on the CMOS wafer. Then slight pressure is applied and the sensor wafer and the CMOS wafer are bonded at temperature $<380^{\circ} \mathrm{C}$ (Fig. $4(\mathrm{~g})$ ). The thick proof-mass is etched on the SOI handle layer by DRIE after wafer bonding. The sensor structure is released by a dry etching the SOI buried oxide layer (Fig. 4(h)).

The fourth part of the process is the sensor micro-package step. The bonded and release etched CMOS-MEMS wafer is encapsulated at wafer-level by a cavity etched silicon cap wafer to protect it from the environment prior to die singulation. The sealed cap also isolates the sensor from the environment as well as the ambient humidity and pressure variations. Finally the capped sensor-circuitry wafer can be singulated into individual sensor die (Fig. 4(i)). Figure 5 is a picture taken from a fully fabricated sensor wafer prior to its final micro-package. An enlarged view of multiple sensor dies is shown, as well.

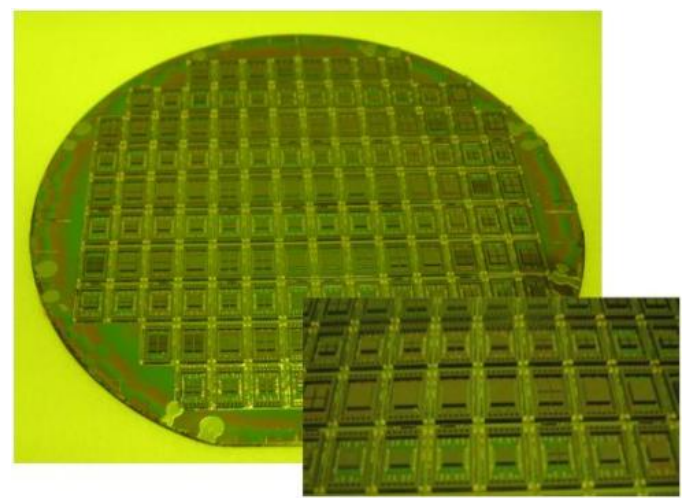

Figure 5: Pictures of a fabricated sensor wafer and a close-up multiple dies prior to the sensor package.

Figure 6 shows the SEM of a single accelerometer after DRIE etching of proof mass from its handle layer. Four sets of sensing fingers can be seen from the figure. The proof mass quadrants are electrically isolated from each other from the device layer side (facing down). They are mechanically connected rigidly through the handle layer as shown in the figure. Figure 7 is a close-up view for a portion of the accelerometer. The sensing fingers, the support beam, one of the proof mass quadrant, and the lead transfer metal pad from fingers attached to the proof mass are all shown in the figure.

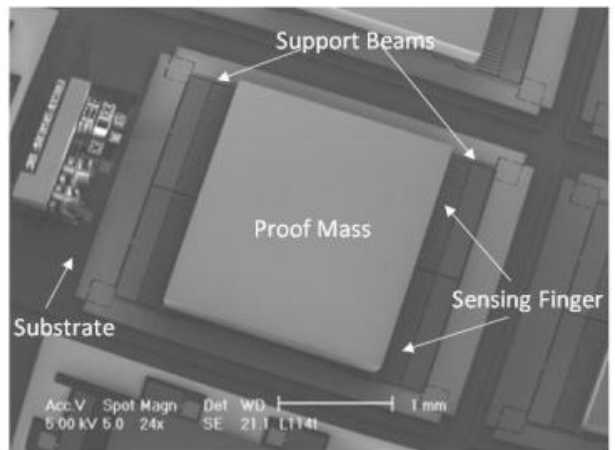

Figure 6: The accelerometer after wafer bonding and proof mass definition.

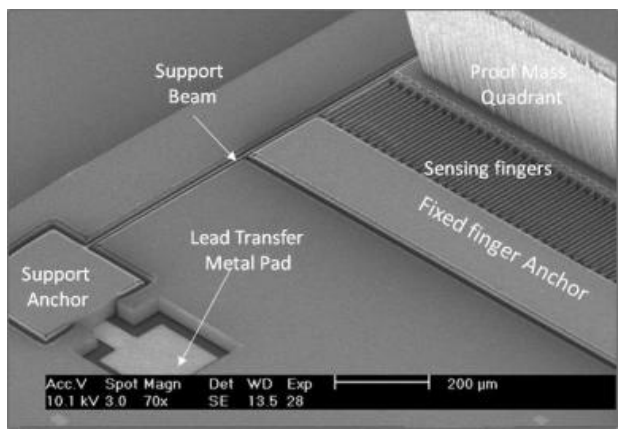

Figure 7: A close-up view of a portion of the fabricated accelerometer. 
The wafer-level micro-packaged CMOS-MEMS accelerometers are shown in Fig. 8. At this step the completed wafer can be diced to individual sensor chip and package it in its housing as needed. Figure 9 presents a single CMOS-MEMS sensor chip that is packaged in standard 64-pin DIP IC housing for testing.

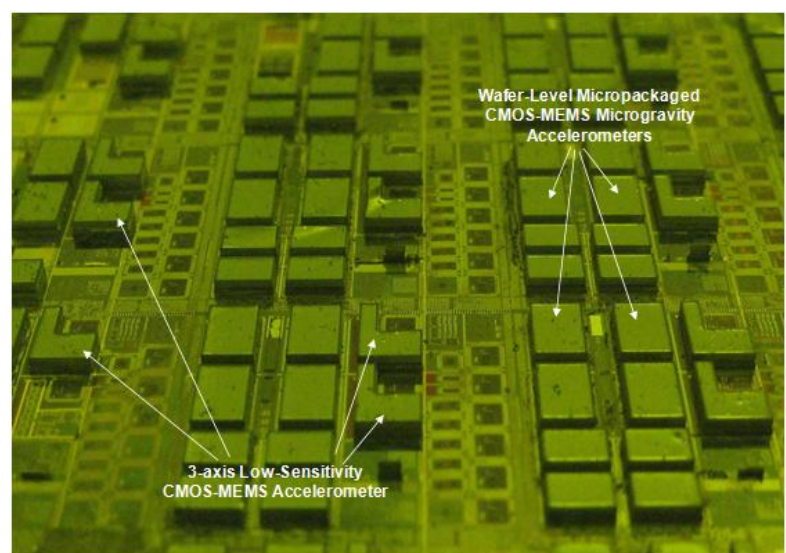

Figure 8: A picture taken from a fully fabricated, integrated and wafer-level micro-packaged CMOS-MEMS accelerometer wafer.

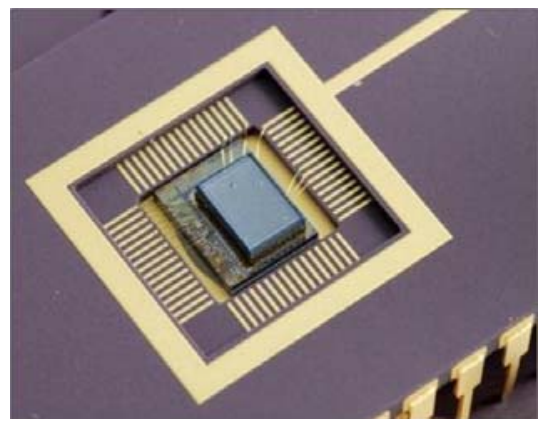

Figure 9: A singulated CMOS-MEMS microgravity accelerometer chip packaged in a standard 64-pin DIP IC housing and wire bonded for testing.

\section{SENSOR MEASUREMENTS}

The fabricated microgravity accelerometers were tested both at the wafer-level by probing and at device level with CMOS interface circuitry. Figure 10(a) presents the measured capacitance response versus a sweeping dc voltage for one set of the sense capacitors. This is a static open-loop test. The measured sensitivity under this condition is $1.7 \mathrm{pF} / \mathrm{g}$ for a single set of sense fingers corresponding to a total sensitivity of $4 \times 1.7 \mathrm{pF} / \mathrm{g}$ from all four sets of interdigitated sense fingers. The noise floor for this accelerometer is $1.2 \mu \mathrm{g} / \mathrm{rt}-\mathrm{Hz}$.

The fabricated and micro-packaged accelerometer was also tested together with its interface CMOS read-out circuit. The sensor chips were mounted and wire bonded in standard 64-pin DIP IC housing as shown in Fig 9. Figure 10(b) is a screen shot showing the measurement result for a single packaged CMOSMEMS accelerometer in turn-over tests. The sensitivity is $1.8 \mathrm{~V} / \mathrm{g}$ with power dissipation of $6 \mathrm{~mW}$ at $3 \mathrm{~V}$.

\section{CONCLUSION}

In conclusion, a single-chip CMOS-MEMS microgravity accelerometer has been designed, fabricated, and tested. This sensor has a fully-symmetrical full-bridge sense capacitor enabling precision readout without any need for reference capacitors. Its single-crystal silicon sensor structure improves long term-stability.
Test results indicate that this MEMS-CMOS microgravity accelerometer has high sensitivity and low thermo-mechanical noise. Because of its high performance and CMOS manufacturing compatibility, this microgravity accelerometer can be readily used in applications demanding precision, including inertial navigation.

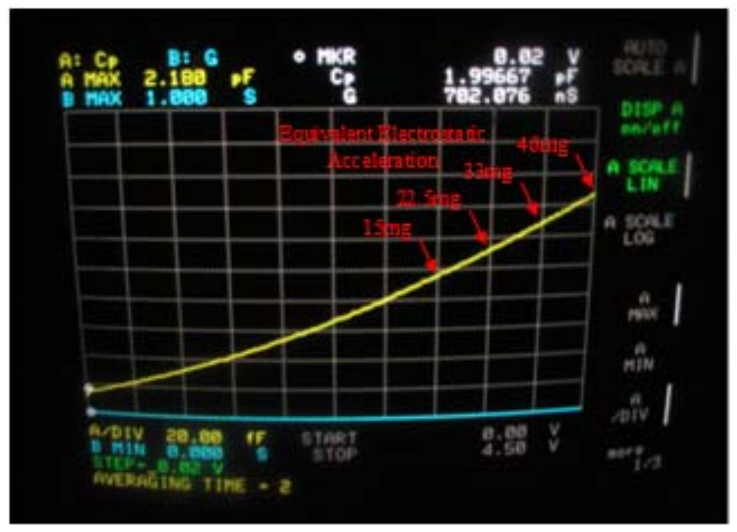

(a)

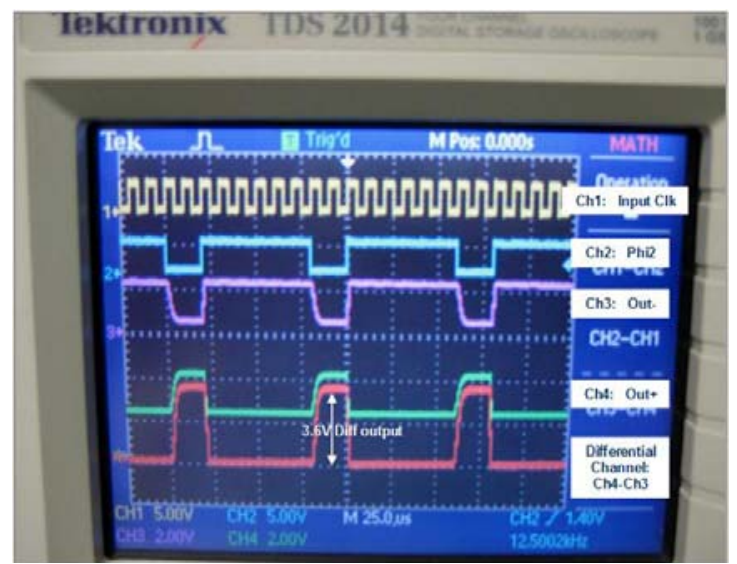

(b)

Figure 10:Measurement results: (a) measured sensitivity of one group sense fingers $4 \times 1.7 \mathrm{pF} / \mathrm{g}$ by sweeping dc voltage and (b) measured sensitivity of $1.8 \mathrm{~V} / \mathrm{g}$ for an integrated CMOS-MEMS sensor using turn-over test.

\section{REFERENCES}

[1] N. Yazdi, A. Ayazi, and K. Najafi, "Micromachined Inertial Sensors", Proceedings of the IEEE, vol. 86, no. 8, pp. 16401659, August 1998.

[2] N. Yazdi, K. Najafi, "An All-Silicon Single-Wafer Micro-g Accelerometer with a Combined Surface and Bulk Micromachining Process," IEEE/ASME Journal of Microelectromechanical Systems (JMEMS), vol. 9, no. 4, December 2000, pp. 544-550.

[3] B. Amini, F. Ayazi, "A 2.5-V 14-bit $\Sigma \Delta$ CMOS SOI capacitive accelerometer", IEEE Journal of Solid-State Circuits (JSSC), v. 39, no. 12, Dec. 2004, pp.2467-2476.

[4] B. E. Boser, R.T. Howe, "Surface Micromachined Accelerometers", IEEE Journal of Solid-State Circuits (JSSC), v. 31, no. 3, Mar. 1996, pp.366-375.

\section{CONTACT}

*Y. Zhang, tel: +1-734-302-1140; yzhang@evigia.com 